\title{
1 An Escherichia coli Chassis for Production of Electrically Conductive Protein Nanowires
}

3 Toshiyuki Ueki ${ }^{1,2^{*}}$, David J.F. Walker ${ }^{1,2^{*}}$, Trevor L. Woodard ${ }^{1}$, Kelly P. Nevin ${ }^{1}$, Stephen S.

4 Nonnenmann ${ }^{2,3}$, and Derek R. Lovley ${ }^{1,2^{*}}$

$6 \quad{ }^{1}$ Department of Microbiology, University of Massachusetts-Amherst, Amherst, MA, USA

$7 \quad{ }^{2}$ Institute for Applied Life Sciences, University of Massachusetts-Amherst

$8 \quad{ }^{3}$ Department of Mechanical and Industrial Engineering, University of Massachusetts-Amherst

$9 *$ Authors contributed equally 


\section{Abstract}

12 Geobacter sulfurreducens' pilin-based electrically conductive protein nanowires (e-PNs) are a

13 revolutionary electronic material. They offer novel options for electronic sensing applications

14 and have the remarkable ability to harvest electrical energy from atmospheric humidity.

15 However, technical constraints limit mass cultivation and genetic manipulation of $G$.

16 sulfurreducens. Therefore, we designed a strain of Escherichia coli to express e-PNs by

17 introducing a plasmid that contained an inducible operon with E. coli genes for type IV pili

18 biogenesis machinery and a synthetic gene designed to yield a peptide monomer that could be

19 assembled into e-PNs. The e-PNs expressed in E. coli, and harvested with a simple filtration

20 method, had the same diameter $(3 \mathrm{~nm})$ and conductance as e-PNs expressed in $G$.

21 sulfurreducens. These results, coupled with the robustness of E. coli for mass cultivation and the

22 extensive E. coli toolbox for genetic manipulation, greatly expands opportunities for large-scale

23 fabrication of novel e-PNs.

25 Keywords: Protein nanowire, e-pili, bioelectronic materials, e-biologics, Geobacter,

26 sustainable electronics 


\section{Introduction}

Electrically conductive protein nanowires (e-PNs) show promise as revolutionary,

30 sustainably produced, and robust electronic materials ${ }^{1-8}$. They are biocompatible and can readily

31 be adapted for a multitude of sensing applications ${ }^{1-7}$. Devices comprised of thin layers of e-PNs

32 function as 'humidity-powered electrical generators', continuously harvesting energy in the form

33 of electricity from atmospheric humidity ${ }^{8}$. However, the implementation of e-PNs in electronic

34 devices has been limited due to a lack of methods for mass production. In vitro assembly of

35 peptides into conductive nanofilaments is feasible ${ }^{9,10}$, but the filaments tend to agglomerate into

36 gels at high peptide concentrations, limiting possibilities for large-scale fabrication. Furthermore,

37 synthesis of the peptide monomers required for in vitro assembly is expensive, potentially

38 limiting e-PN affordability.

In vivo assembly of e-PNs with microorganisms has several advantages over in vitro

40 synthesis. Benefits include much lower cost and greater flexibility in e-PN design options with a

41 production platform fueled with inexpensive, renewable feedstocks. A diversity of bacteria and

42 archaea assemble peptides that show homology to bacterial type IV pilins into e-PNs ${ }^{11-15}$, but the

43 e-PNs of Geobacter sulfurreducens have been most intensively investigated 1, $16 . G$.

44 sulfurreducens e-PNs can be fabricated in vivo with acetate as the carbon and energy source.

45 Once the cells are grown, the e-PNs can be harvested, retaining their conductive properties ${ }^{12,17-}$ $46 \quad 19$.

47 The exquisite machinery that bacteria possess to assemble pilin proteins into filaments 20 48 confers great control over e-PN production, yielding a highly uniform product. The microbial 49 assembly process also offers substantial opportunities for producing diverse, new types of e-PNs.

50 For example, the conductivity of e-PNs produced with G. sulfurreducens has been tuned over a 
51 million-fold with simple modifications to the G. sulfurreducens pilin gene to either increase or

52 decrease the abundance of aromatic amino acids ${ }^{1,16}$. Pilin genes can be designed to encode

53 additional peptides at the carboxyl end of the pilin, yielding e-PNs that retain their conductivity

54 and display the added peptides on the outer surface of the wires ${ }^{7}$. This peptide display along the

55 wires offers unique possibilities for introducing peptide ligands to confer specific sensing

56 functions to e-PN devices with a flexibility in sensor design not feasible with other materials

57 such as carbon nanotubes or silicon nanowires ${ }^{7}$. Peptides displayed on the outer surface of e-

58 PNs might also be designed to promote e-PN binding to surfaces to facilitate wire alignment or

59 to function as chemical linkers with polymers for the fabrication of composite materials ${ }^{7}$.

60 Synthetic gene circuits introduced to control the expression of multiple e-PN monomer genes

61 within a single cell offer the possibility to further tune e-PN function by producing

62 heterogeneous wires comprised of multiple types of e-PN monomers with the stoichiometry of

63 each monomer type precisely controlled ${ }^{7}$. These design options would be difficult to replicate

64 with in vitro assembly of e-PNs or fabrication of nanowires from non-biological materials.

65 Geobacter-fabricated e-PNs have several other advantages over traditional non-biological

66 nanowire materials. Production of the e-PNs requires 100 -fold less energy than is required for

67 fabricating silicon nanowires or carbon nanotubes ${ }^{2}$. No toxic chemicals are required for e-PN

68 fabrication and the final product is biocompatible, environmentally benign, and recyclable ${ }^{2}$. Yet,

69 e-PNs are remarkably robust, maintaining function even under harsh CMOS-compatible

70 fabrication conditions ${ }^{6}$. Proof-of-concept studies have demonstrated the dynamic sensing

71 response of Geobacter-fabricated e-PNs; the ability of these e-PNs to function as the conductive

72 component in flexible electronics; and that, in the appropriate electrode/e-PN configurations, thin

73 films of Geobacter e-PNs can generate electricity from the humidity naturally present in air 6-8, 
75 Barriers to large-scale e-PN production have been a limitation to realizing the potential of

76 Geobacter e-PNs for these and other possible applications. G. sulfurreducens must be grown

77 anaerobically to produce e-PNs. This requirement adds technical complexity and costs. A strain

78 of Pseduomonas aeruginosa, grown aerobically, heterologously expressed the G. sulfurreducens

79 pilin gene with the assembly of e-PNs with properties similar to the e-PNs expressed in $G$.

80 sulfurreducens ${ }^{21}$. However, $P$. aeruginosa is a pathogenic microorganism and thus not ideal for

81 large-scale commercial production of e-PNs. Furthermore, the expression of the e-PNs in $P$.

82 aeruginosa remained under the control of the native regulatory system, limiting options for

83 controlling the timing and extent of e-PN expression ${ }^{21}$.

84 We thought that Escherichia coli might be an ideal chassis for e-PN fabrication. E. coli is

85 a common platform for the commercial scale production of organic commodities ${ }^{22,23}$. The

86 substantial E. coli genetic toolbox, including the possibility of introducing unnatural amino acids

$87^{24,25}$, could provide broad options for designing e-PNs with different properties and functions.

88 Non-pathogenic strains of E. coli typically do not express type IV pili. However, introduction of

89 an artificial operon of pilus assembly protein genes from pathogenic E. coli into non-pathogenic

90 E. coli yielded a non-pathogenic strain that expressed the same type IV pili that pathogenic $E$.

91 coli express ${ }^{26}$. This finding, and the fact that bacteria will often assemble heterologous pilins

92 into pili $12,13,21,27-29$, suggested that it might be possible to develop a non-pathogenic strain of $E$.

93 coli that would express Geobacter e-PNs.

94 Another limitation to large-scale in vivo production of e-PNs has been the methods for

95 separating the e-PNs from cells. Previously described methods have included multiple laborious 
96 steps, often with strategies such as ultracentrifugation and/or salt precipitation procedures that

97 would be difficult to economically scale ${ }^{12,17}$.

98 Here we report on the construction of a strain of E. coli amended with genes for the

99 expression of type IV pili assembly machinery and a synthetic gene designed to yield e-PNs

100 comparable to those produced by G. sulfurreducens. This strain produces e-PNs with

101 characteristics similar to the e-PNs expressed by G. sulfurreducens. Simple aerobic growth of the

102 E. coli designed for e-PN production, coupled with a new simplified method for harvesting e-

103 PNs from cells, suggests that large-scale production of e-PNs will be feasible.

104

\section{Results and Discussion}

We engineered a standard lab strain of E. coli to produce e-PNs by introducing the genes

107 known from previous studies ${ }^{26}$ to be required for type IV pili biogenesis. We modified the

108 previous design of $E$. coli $^{26}$, which was constructed to produce pili from the $E$. coli pilin protein

109 PpdD, as follows: 1) the gene for pilin monomer is within a separate cloning site to make it

110 convenient to exchange the gene for the pilin of interest (Figure 1a); 2) the gene clusters were

111 constructed with common and less expensive restriction enzymes to aid in modularity (Figure

$1121 \mathrm{~b})$; 3) ribosome binding sites for $\operatorname{hof} B$, hofM, and $p p d A$ were changed to improve translation

113 efficiency (Figure 1c); 4) a gene cluster containing $p p d A, p p d B, y g d B$, and $p p d C$ and $g s p O$ were

114 connected by 2-step PCR instead of using a restriction enzyme to delete unnecessary sequence

115 (Figure 1b,d); 5) intergenic regions were shortened to delete unnecessary sequence (Figure 1d);

116 6) the tac promoter ${ }^{30}$, one of the strongest promoters in E. coli, was incorporated to enhance

117 transcription of the genes for the assembly of type IV pili (Figure 1b); and 7) a gene for the LacI

118 repressor was included in the expression vector to repress the genes when desired, such as during 
119 cloning (Figure 1a). We also deleted the gene fimA to prevent the formation of type I pili using

120 previously described genetic methods 31,32 .

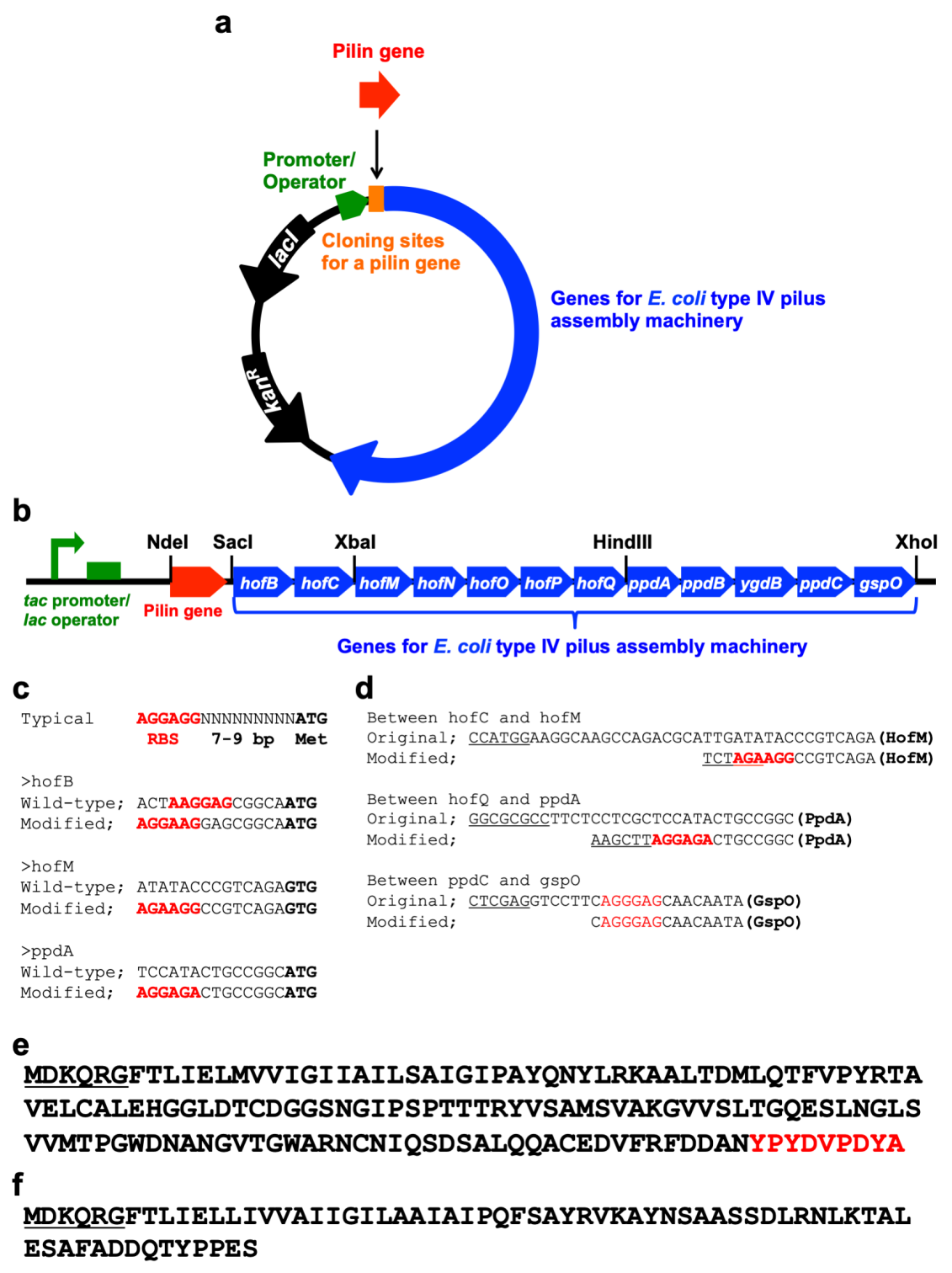

122 Figure 1. Construction of type IV pilus assembly system in E. coli and synthetic peptide 123 monomer for e-PN assembly. (a) Expression vector for genes for type IV pilus assembly 124 in E. coli. (lacI, Lac repressor gene; $k a n^{R}$, kanamycin resistance gene); (b) Gene 125 organization for E. coli type IV pilus assembly with cloning sites designated; (c) 126 Ribosome binding sites (designated in red) that were changed from those in previous 
studies ${ }^{26}$; (d) Intergenic regions in the synthetic operon that were changed from those in previous studies ${ }^{26}$ (ribosome binding sites in red, restriction enzyme sites underlined); (d) Amino acid sequence of the HA-tagged PpdD ( signal sequence underlined, HA tag sequence in red); (f) Amino acid sequence of the synthetic peptide monomer designed for assembly in e-PNs, which is a combination of a portion of the G. sulfurreducens pilin, PilA, with the E. coli PpdD signal sequence (underlined). 


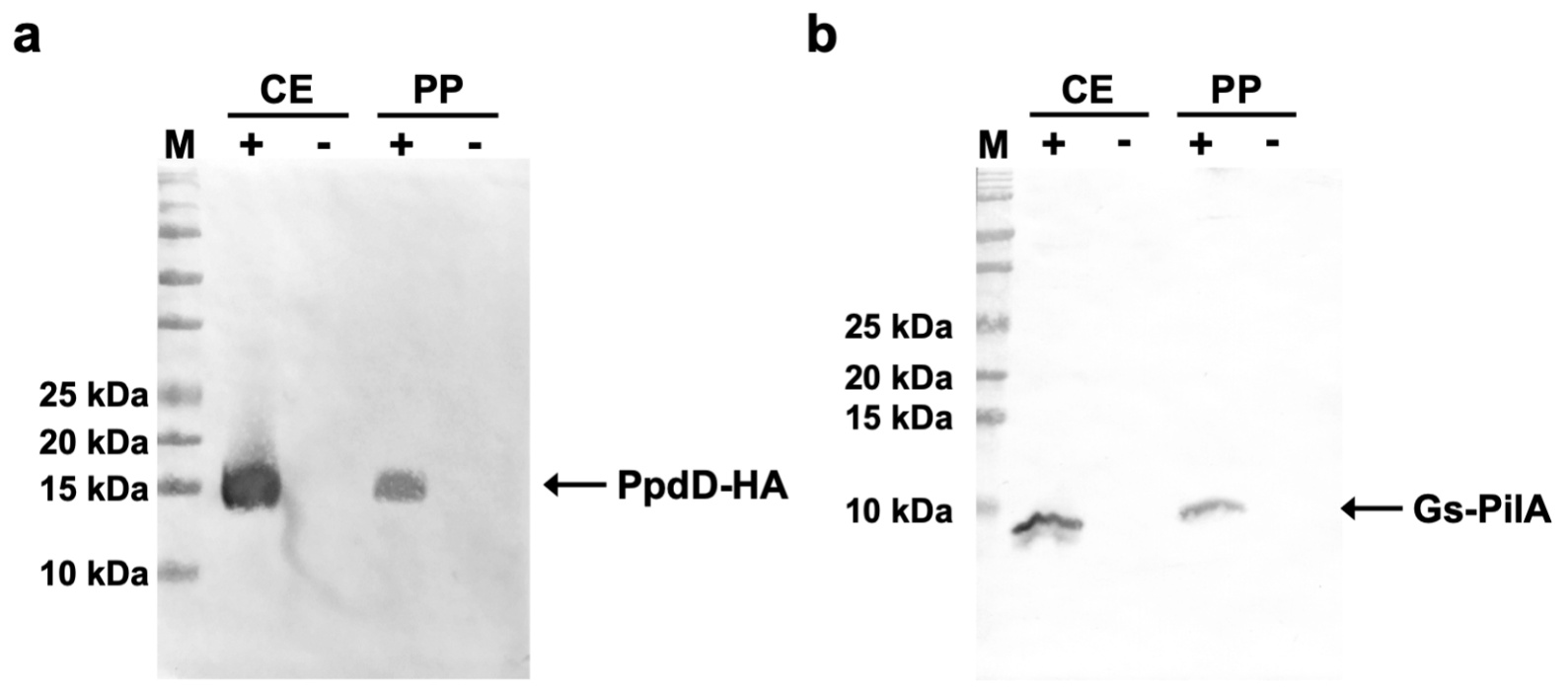

Figure 2. Expression of pili and e-PNs in E. coli. (a) Results with the strain of E. coli with genes for pilus assembly and the gene for PpdD-HA. (b) Results from E. coli strain GPN, which contained genes for pilus assembly and the gene for a synthetic pilin monomer designed to yield e-PNs assembled from a modified G. sulfurreducens PilA monomer. Western blot analyses of denatured proteins separated on an SDS PAGE gel and analyzed with antibody for (a) the HA tag on PpdD-HA pilin or (b) G. sulfurreducens PilA. Strains with the pilin genes designated with $(+)$. Control strains without the pilin genes designated (-). Samples from whole cell extracts (CE) and the pili preparations To express e-PNs similar to those expressed in G. sulfurreducens in E. coli, we designed 156 a gene to yield a synthetic peptide monomer for assembly into e-PNs. The peptide was similar to 157 the G. sulfurreducens pilin monomer, PilA, with the exception that the signal sequence was 158 replaced with the $E$. coli PpdD signal sequence to facilitate e-PN assembly in $E$. coli (Figure 1f). 159 The gene for the synthetic e-PN monomer was cloned into the location designated 'pilin gene' 160 (Figure 1a). The strain with the synthetic gene for the e-PN monomer was designated E. coli 
161 strain GPN (Geobacter protein nanowire). The e-PN monomer was detected in whole cell

162 extracts of strain GPN with PilA antibody, but not in the control strain that lacked the gene for

163 the e-PN monomer (Figure 2b).

164 e-PNs were harvested from strain GPN with physical shearing from the cells, as in

165 previous studies of e-PNs expressed in G. sulfurreducens ${ }^{17}$. In those previous studies, the cells

166 were separated from the sheared e-PNs with centrifugation and then the e-PNs in the supernatant

167 were collected with ultracentrifugation or ammonium sulfate precipitation ${ }^{17}$. These methods of

168 e-PN collection are labor intensive and will be difficult to adapt to large-scale production.

169 Therefore, the e-PNs sheared from strain GPN and separated from cells were treated with Triton

$170 \mathrm{X} 100$ detergent and then were collected on a filter with a $100 \mathrm{kDa}$ molecular weight cutoff. This

171 method is simpler and faster than previously described ${ }^{17}$ e-PN purification methods.

172 The e-PNs harvested from E. coli strain GPN were ca. $3 \mathrm{~nm}$ in diameter and several $\mu \mathrm{m}$

173 in length (Figure 3a), a morphology similar to the e-PNs expressed in G. sulfurreducens. No

174 filaments were observed in similar preparations when the gene for the e-PN monomer was

175 omitted from E. coli strain GPN. Denaturation of the e-PNs from E. coli strain GPN yielded a

176 monomer that reacted with PilA antibody whereas the monomer was not detected in preparations

177 from the control strain without the gene for the e-PN monomer (Figure $2 b$ ).

178 The conductance of thin films of the e-PNs from E. coli strain GPN, determined with a 179 nanoelectrode array as previously described ${ }^{13}$, was $3.26 \pm 0.35 \mu \mathrm{S}$, similar to the conductance of $1803.39 \pm 0.04 \mu \mathrm{S}$ for e-PNs harvested from G. sulfurreducens (Figure 3b). The conductance of 181 these e-PNs was much higher than the conductance of wires harvested from strain Aro-5 (Figure $1823 \mathrm{~b}$ ), a strain of $G$. sulfurreducens that expresses a synthetic pilin gene designed to yield protein 183 nanowires with low conductivity ${ }^{18,27}$. 


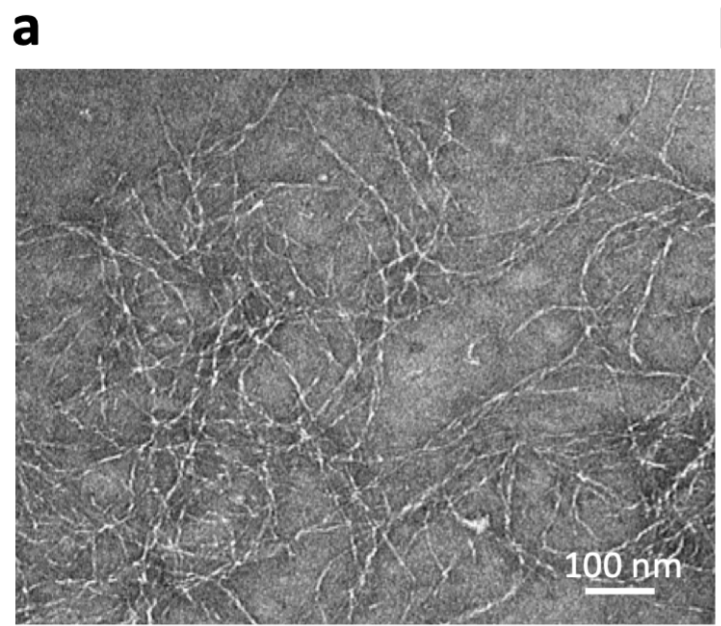

184 published previously ${ }^{13}$. individual e-PNs expressed in G. sulfurreducens.

\section{b}

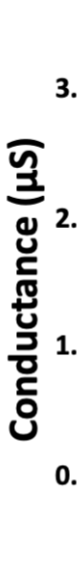

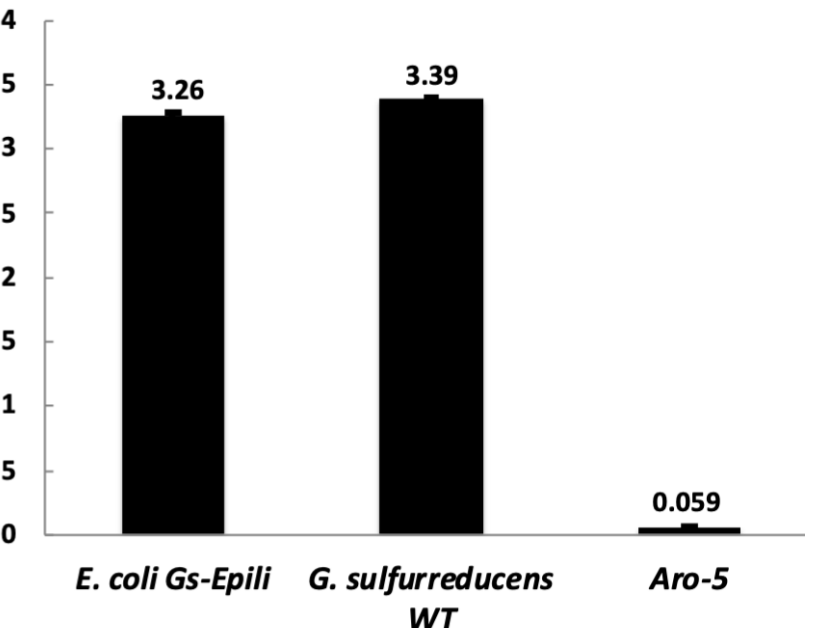
WT

Figure 3. Characterization of the e-PNs expressed in E. coli strain GPN. (a) Transmission electron micrograph. (b) Conductance of films of e-PNs expressed in $E$. coli strain GPN compared with e-PNs from wild-type Geobacter sulfurreducens and the Aro-5 strain of G. sulfurreducens. The results are the means and standard deviation of triplicate measurements on each nanoelectrode array, and at least three independent nanoelectrode arrays. Results for wild-type G. sulfurreducens and strain Aro-5 were

The conductance of individual e-PNs was evaluated on highly oriented pyrolytic graphite with atomic force microscopy employing a conductive tip, as previously described ${ }^{15}$. The diameter of the e-PNs was $3 \pm 0.04 \mathrm{~nm}(\mathrm{n}=18 ; 6$ measurements on 3 independent pili) the same as e-PNs expressed with G. sulfurreducens (Figure 4). The e-PNs were conductive with an ohmic-like current-voltage response (Figure 4d). The conductance of the individual e-PNs, $4.3 \pm$ $0.8 \mathrm{nS}(\mathrm{n}=9)$, compared well with the previously reported ${ }^{15}$ conductance of $4.5 \pm 0.3 \mathrm{nS}$ for 


\section{a}
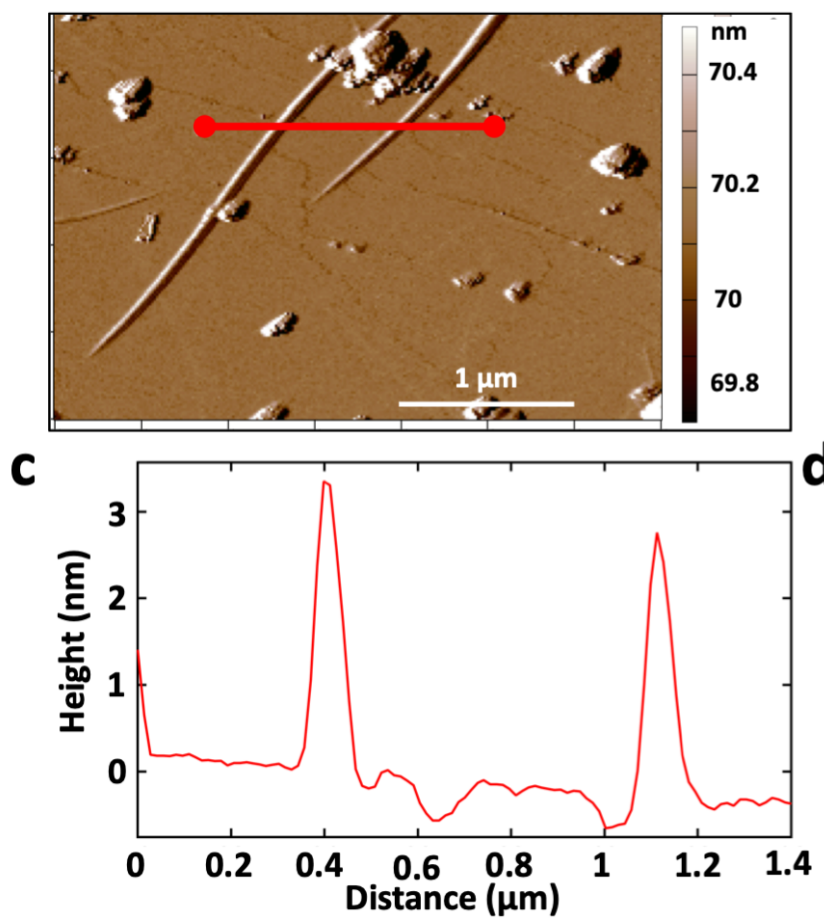

b
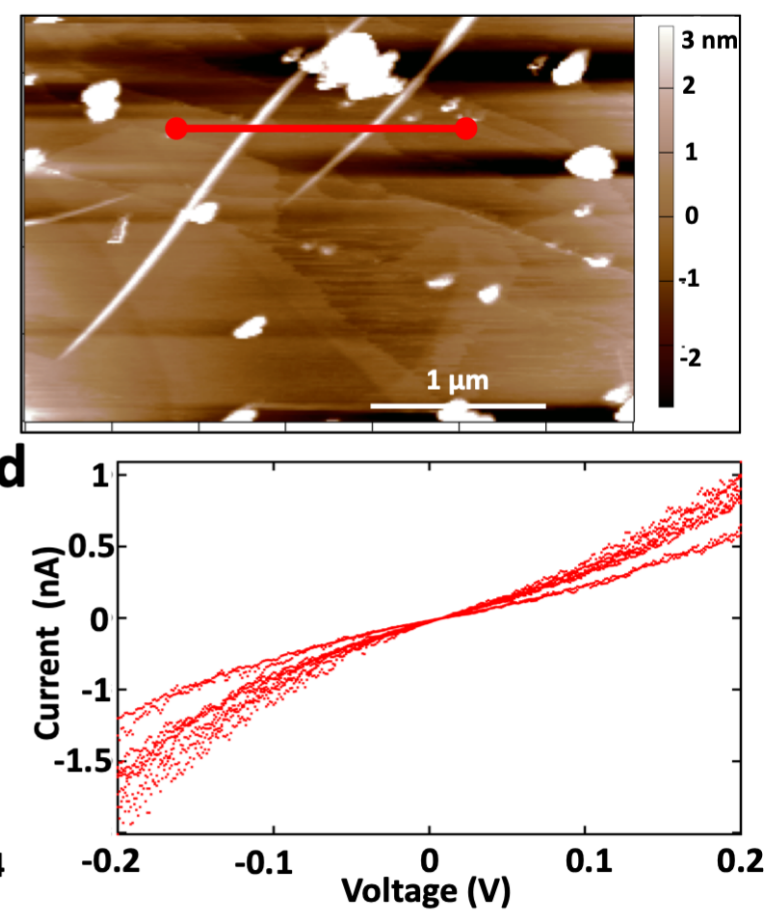

Figure 4. Characterization of individual e-PNs expressed in E. coli strain GPN. (a) Amplitude image of 2 e-PNs in amplitude modulation mode (b) Height image of each e-PN (c) Cross-section line trace showing the height of 2 individual e-PNs, designated in panel a and $\mathrm{b}$ by the redline, demonstrating $\sim 3 \mathrm{~nm}$ height (diameter).

(d) Current voltage response of nine individual measurements ( 3 measurements on 3 e-PNs).

\section{Conclusions}

The fabrication of e-PNs with E. coli offers substantial advantages over expression in $G$.

209 sulfurreducens. Special equipment and expertise is required to anaerobically culture $G$.

210 sulfurreducens, whereas $E$. coli can be simply grown under ambient, aerobic atmospheric

211 conditions. When coupled with our finding that the e-PNs can be collected with simple filtration

212 method, expression of e-PNs in E. coli offers the potential for large-scale e-PN production. 
214 wider diversity of e-PNs than would currently be possible with G. sulfurreducens. Tools for the 215 genetic manipulation of $G$. sulfurreducens are limited and only the most simple synthetic gene 216 circuits have been adapted for this organism ${ }^{33}$. The much broader range of strategies for

217 introducing genes and controlling their expression in E. coli ${ }^{22,23}$ is expected to enable the design

218 and expression of e-PNs with unique properties and functionalities that could not readily be 219 fabricated with G. sulfurreducens. Most notably, E. coli is an excellent platform for modifying 220 proteins with unnatural amino acids that can confer diverse new functionalities to proteins ${ }^{24,25}$. 221 The development of new e-PNs in the E. coli chassis for enhanced sensor, electronics, and 222 energy-harvesting applications ${ }^{1-8}$ is underway.

224 Materials and Methods

\section{$225 E$. coli strain and culture conditions}

E. coli NEB 10-beta (New England Biolabs) was grown at $37{ }^{\circ} \mathrm{C}$ in $\mathrm{LB}$ medium

227 supplemented with appropriate antibiotics as necessary for plasmid preparation, as previously

228 described ${ }^{34}$. The gene for FimA, the primary monomer for type I pili, was deleted as previously

229 described ${ }^{31,32}$ to construct E. coli $\Delta$ fimA (kanamycin sensitive). The strains expressing the

230 modified E. coli pilin or the synthetic peptide for assembly into e-PNs were built in this strain.

231 Construction of expression vector for type IV pilus assembly

An expression vector for type IV pilus assembly was constructed as described previously $233{ }^{26}$ with several modifications (Figure 1). To construct the basic expression vector for type IV 234 pilus assembly the $\mathrm{T} 7$ promoter in the plasmid vector pET24b (Novagen) was replaced with tac 235 promoter ${ }^{30}$. The DNA fragment containing tac promoter and lac operator was amplified by PCR 
with a primer pair, Ptac-F/Olac-R (Table S1) and pCD341 ${ }^{35}$ as template. The PCR product was digested with BglII and $\mathrm{XbaI}$ and replaced the $\mathrm{Bg} 1 \mathrm{II}-\mathrm{XbaI}$ region containing $\mathrm{T} 7$ promoter and lac operator in $\mathrm{pET} 24 \mathrm{~b}$. The resultant plasmid was designated $\mathrm{p} 24 \mathrm{Ptac}$.

Next, genes for E. coli type IV pilus assembly without the major pilin gene were cloned in p24Ptac. The genes include hofB (ATPase), hofC (platform protein), hofM (assembly protein),

241 hofN (assembly protein), hofO (assembly protein), hofP (assembly protein), hofQ (secretin), $p p d A$ (minor pilin), $p p d B$ (minor pilin), $y g d B$ (minor pilin), $p p d C$ (minor pilin), and $g s p O$

243 (prepilin peptidase) ${ }^{26}$. DNA fragment containing $p p d A, p p d B, y g d B$, ppdC, and gspO was

244 prepared by 2-step PCR. Fragments containing $p p d A, p p d B, y g d B$, and $p p d C$, or $g s p O$ were 245 amplified by PCR with primer pairs, ppdA-F/ppdC-R and gspO-F/gspO-R (Table S1), 246 respectively. The fragment containing $p p d A, p p d B, y g d B$, $p p d C$, and $g s p O$ was amplified by PCR 247 with these PCR products as template and a primer pair, ppdA-F/gspO-R. The PCR product was 248 digested with HindIII and XhoI and cloned in pBluescript II SK (Stratagene). The fragment 249 containing $h \circ f M$, hof $N$, hofO, hofP, and $h \circ f Q$ was amplified by PCR with the primer pair, hofM250 F/ hofQ-R (Table S1). The PCR product was digested with XbaI and HindIII and cloned in the 251 plasmid containing $p p d A, p p d B, y g d B, p p d C$, and gspO. The fragment containing hofB and hofC 252 was amplified by PCR with a primer pair, hofB-F/hofC-R (Table S1). The PCR product was 253 digested with SacI and XbaI and cloned in the plasmid containing hofM, hof $N$, hofO, hofP, hofQ, $254 \operatorname{ppd} A, p p d B, y g d B, p p d C$, and gspO. Fragment containing hofB, hofC, hofM, hofN, hofO, hofP, $255 h o f Q, p p d A, p p d B, y g d B, p p d C$, and $g s p O$ was prepared by digesting the plasmid containing 256 hofB, hofC, hofM, hofN, hofO, hofP, hofQ, $p p d A, p p d B, y g d B$, ppdC, and gspO with SacI and 257 XhoI and cloned in p24Ptac (Figure 1). The resultant plasmid was designated T4PAS/p24Ptac. 
The fragment containing a gene for PpdD with HA tag (Figure 1e) was amplified with a 260 primer pair, ppdD-F/ppdD-HA-R (Table S1), digested with NdeI and SacI, and cloned in

261 T4PAS/p24Ptac. The resultant plasmid was termed ppdD-HA/T4PAS/p24Ptac.

262 For initial studies with the E. coli strain expressing the E. coli pilin PpdD, the plasmids ppdD-

$263 \mathrm{HA} / \mathrm{T} 4 \mathrm{PAS} / \mathrm{p} 24 \mathrm{Ptac}$ or T4PAS/p24Ptac were transformed into E. coli $\Delta$ fimA. A single colony

264 from LB agar plate containing kanamycin ${ }^{34}$ was inoculated in TB medium (Novagen)

265 supplemented with $1 \%$ glycerol and kanamycin and incubated at $30^{\circ} \mathrm{C}$ for $24 \mathrm{~h}$ to the stationary

266 phase. Pili were sheared from cells and precipitated with TCA as described previously ${ }^{26}$.

\section{Expression and harvesting of e-PNs}

A fragment encoding a gene for a synthetic pilin monomer, which was similar to the PilA

269 monomer of G. sulfurreducens but included the signal sequence of PpdD instead of the original

270 PilA signal sequence (Figure 1f), was amplified with a primer pair, EPS-GspilA-F/GspilA-R

271 (Table S1). The amplified fragment was digested with NdeI and SacI and cloned in

272 T4PAS/p24Ptac. The resultant plasmid, designated GspilA/T4PAS/p24Ptac, was transformed

273 into E. coli $\triangle$ fimA. The resultant strain, designated E. coli strain GPN ( $\underline{\text { Geobacter protein }}$

274 nanowires) was grown on $10 \mathrm{~cm}$ diameter culture plates of standard LB medium ${ }^{34}$ amended with

275 kanamycin, and solidified with agar. After overnight growth at $30^{\circ} \mathrm{C}$, cells were scraped from

276 the surface and suspended in $6 \mathrm{ml}$ of M9 media ${ }^{34}$. Twenty plates of M9 medium supplemented

277 with $0.5 \%$ glycerol, $0.5 \mathrm{mM}$ IPTG, and kanamycin were spread-plated with $300 \mu$ of the

278 suspended cells. The plates were incubated at $30{ }^{\circ} \mathrm{C}$ for 48 hours. Cells were harvested from the 279 plates with $1 \mathrm{ml}$ of M9 media (500 $\mu 1$ to scrape, $500 \mu 1$ to wash) for each plate. The $20 \mathrm{ml}$

280 suspension of cell scrapings was centrifuged at $4000 \mathrm{rpm}$ for 15 minutes at $4{ }^{\circ} \mathrm{C}$ to pellet the 281 cells. The supernatant was discarded and the cells were resuspended in $30 \mathrm{ml}$ of $150 \mathrm{mM}$ 
282 ethanolamine $(\mathrm{pH}$ 10.5) buffer and poured into a Waring blender. The tubes were washed three

283 times with $20 \mathrm{ml}$ of the ethanolamine buffer, which was also added to the blender. The $90 \mathrm{ml}$

284 suspension was blended for 2 minutes on low speed. The contents of the blender were transferred

285 to a centrifuge bottle along with a wash of the blender with $10 \mathrm{ml}$ of ethanolamine buffer. The

286 blended material was centrifuged at $5000 \mathrm{x} \mathrm{g}$ for 30 minutes at $4^{\circ} \mathrm{C}$. The supernatant was

287 collected. Triton X100 detergent was added to provide a final concentration of $6 \mathrm{mM}$. The

288 mixture was shaken at $100 \mathrm{rpm}$ at room temperature for 45 minutes then added to a stirring

289 filtration unit that had a $100 \mathrm{kDa}$ molecular weight cutoff membrane filter made from

290 polyethersulfone (Omega membrane 100K $76 \mathrm{~mm}$, Pall Corporation). Additional ethanolamine

291 buffer was added to dilute the sample to yield a final Triton X100 concentration of $2 \mathrm{mM}$. The

292 sample was filtered under nitrogen gas $(69 \mathrm{kPa})$. The sample on the filter was washed four times

293 with $100 \mathrm{ml}$ of water. The e-PNs were collected from the filter by scrapping the surface into 500

$294 \mu \mathrm{l}$ of water. The scrapping procedure was repeated two more times to yield a suspension of e-

295 PNs in $1.5 \mathrm{ml}$ of water.

296 Western blot analysis

297 The presence of pilin monomers in whole cell extracts and pili preparations was 298 evaluated with Western blot analysis. Whole cell extracts were prepared with B-PER Complete

299 Bacterial Protein Extraction Reagent (Thermo Fisher Scientific). Western blot analysis was

300 conducted as described previously ${ }^{7}$. PpdD-HA pilin was detected with an anti-HA antibody (HA

301 Tag Polyclonal Antibody, Invitrogen). The G. sulfurreducens pilin monomer, PilA, was detected 302 with an anti-PilA antibody ${ }^{7}$.

303 Protein Nanowire Conductance 
The conductance of e-PNs expressed in E. coli from the G. sulfurreducens pilin was analyzed as previously described ${ }^{13,15}$. The e-PN preparation in water was adjusted to $500 \mu \mathrm{g}$ protein per $\mu$ l. As previously described ${ }^{13}$, a $2 \mu$ aliquot of the e-PN preparation was dropcast

307 onto the center of three different gold electrode nanoarrays and allowed to dry for 1 hour at 24

$308{ }^{\circ} \mathrm{C}$, after which another $2 \mu \mathrm{l}$ was dropcast and left to dry overnight at $24{ }^{\circ} \mathrm{C}$. Each of the three 309 electrodes nanoarrays was analyzed with a Keithley 4200 Semiconductor Characterization 310 System setup with four probes to conduct a current-voltage (I-V) curve using a $\pm 30 \times 10^{-8} \mathrm{~V}$ 311 sweep with a 5s delay and a 250s hold time. The analyses on each of the three nanoarrays were 312 repeated in triplicate. Thin film conductance was calculated by extracting the slope of the linear 313 fit of the current-voltage response for each of the three measurements on the three electrodes 314 using the formula; $\mathrm{G}=\mathrm{I} / \mathrm{V}$, where $G$ is the conductance, $I$ is the current and $V$ is the voltage. In order to evaluate the conductance of individual e-PNs, $100 \mu$ l aliquot of a culture of $E$.

316 coli expressing the G. sulfurreducens pilin was dropcast onto highly oriented pyrolytic graphite 317 (HOPG) and allowed to sit for 10 minutes. Then the excess liquid was wicked away with a 318 Kimwipe, and an equal volume of deionized water was added to wash off excess salts etc., 319 blotted dry, then dried for 12 hours at $24{ }^{\circ} \mathrm{C}$. Samples were loaded into an Oxford Instruments 320 Cypher ES Environmental AFM and equilibrated for at least 2 hours. The AFM was operated in 321 ORCA electrical mode with a Pt/Ir- coated Arrow-ContPT tip with a $0.2 \mathrm{~N} / \mathrm{m}$ force constant 322 (NanoWorld AG, Neuchâtel, Switzerland). e-PNs were identified in Amplitude Mapping mode 323 (AM-AFM). Point mode current-voltage spectroscopy was carried out by switching to contact 324 mode and gently touching the conductive tip, which was acting as a translatable top electrode, to 325 the top of the e-PN with a force of $1 \mathrm{nN}$. A voltage sweep of $\pm 0.6 \mathrm{~V}$ set at $0.99 \mathrm{~Hz}$ was applied 
to three independent points on each of three individual e-PNs. The conductance was calculated,

as above, from the slope of the linear fit of the current-voltage response between -0.2 and $0.2 \mathrm{~V}$.

\section{References}

329

1. Lovley, D. R., Electrically conductive pili: biological function and potential applications in electronics. Curr. Opin. Electrochem. 2017, 4, 190-198.

2. Lovley, D. R., e-Biologics: Fabrication of sustainable electronics with 'green' biological materials. mBio 2017, 8, e00695-17.

3. Gutermann, T.; Gazit, E., Toward peptide-based bioelectronics: reductionist design of conductive pili mimetics. Bioelectronics in Medicine 2018, 1, 131-137.

4. $\quad$ Creasey, R. C. G.; Mostert, A. B.; Nguyen, T. A. H.; Virdis, B.; Freguia, S.; Laycock, B., Microbial nanowires -electron transport and the role of synthetic analogues Acta Biomater. 2018, 69, 1-30.

5. Ing, N. L.; El-Naggar, M. Y.; Hochbaum, A. I., Going the distance: long-range conductivity in protein and peptide bioelectronic materials. J. Phys. Chem. B 2018, 122, 104310423.

6. $\quad$ Sun, Y.-L.; Tang, H.-.-Y.; Ribbe, A.; Duzhko, V.; Woodard, T. L.; Ward, J. E.; Nevin, K. P.; Nonnenmann, S.; Russell, T. P.; Emrick, T.; Lovley, D. R., Conductive composite materials fabricated with microbially produced protein nanowires. Small 2018, 14, 1802624 .

7. Ueki, T.; Walker, D. J. F.; Tremblay, P.-L.; Nevin, K. P.; Ward, J. E.; Woodard, T. L.; Nonnenmann, S. S.; Lovley, D. R., Decorating the outer surface of microbially produced protein nanowires with peptides. ACS Synthetic Biology 2019, 8, 1809-1817.

8. Liu, X.; Gao, H.; Ward, J. E.; Liu, X.; Yin, B.; Fu, T.; Chen, J.; Lovley, D. R.; Yao, J., Sustained electric power generation from ambient humidity (manuscript submitted).

9. Creasey, R. C. G.; Shingaya, Y.; Nakayama, T., Improved electrical conductance through self-assembly of bioinspired peptides into nanoscale fibers. Materials Chemistry and Physics 2015, 158, 52-59.

10. Ing, N. L.; Spencer, R. K.; Luong, S. H.; Nguyen, H. D.; Hochbaum, A. I., Electronic conductivity in biomimetic $\alpha$-helical peptide nanofibers and gels. ACS Nano 2018, 12, 26522661.

11. Reguera, G.; McCarthy, K. D.; Mehta, T.; Nicoll, J. S.; Tuominen, M. T.; Lovley, D. R., Extracellular electron transfer via microbial nanowires. Nature 2005, 435, 1098-1101.

12. Tan, Y.; Adhikari, R. Y.; Malvankar, N. S.; Ward, J. E.; Woodard, T. L.; Nevin, K. P.; Lovley, D. R., Expressing the Geobacter metallireducens PilA in Geobacter sulfurreducens yields pili with exceptional conductivity. mBio 2017, 8, e02203-16.

13. Walker, D. J. F.; Adhikari, R. Y.; Holmes, D. E.; Ward, J. E.; Woodard, T. L.; Nevin, K. P.; Lovley, D. R., Electrically conductive pili from genes of phylogenetically diverse microorganisms. ISME J. 2018, 12, 48-58.

14. Walker, D. J. F.; Nevin, K. P.; Nonnenmann, S. S.; Holmes, D. E.; Woodard, T. L.; Ward, J. E.; Rotaru, A.-E.; McInerney, M. J.; Lovley, D. R., Syntrophus conductive pili demonstrate that common hydrogen-donating syntrophs can have a direct electron transfer option bioRxiv 2018, https://www.biorxiv.org/content/10.1101/479683v2. 
15. Walker, D. J. F.; Martz, E.; Holmes, D. E.; Zhou, Z.; Nonnenmann, S. S.; Lovley, D. R., The archaellum of Methanospirillum hungatei is electrically conductive. mBio 2019, 10, e00579-19. 16. Lovley, D. R.; Walker, D. J. F., Geobacter protein nanowires. Frontiers in microbiology 2019, 10, 2078. Inoue, K.; Mester, T.; Covalla, S. F.; Johnson, J. P.; Rotello, V. M.; Tuominen, M. T.; Lovley, D. R., Tunable metallic-like conductivity in nanostructured biofilms comprised of microbial nanowires. Nature Nanotechnology 2011, 6, 573-579. individual Geobacter pili. RSC Advances 2016, 6, 8354-8357. Nevin, K. P.; Xia, Q.; Tuominen, M. T.; Lovley, D. R., Synthetic biological protein nanowires with high conductivity. Small 2016, 12, 4481-4485. biogenesis in Gram-negative bacteria. Nature Reviews Microbiology 2017, 15, 365-379.

21. Liu, X.; Wang, S.; Xu, A.; Zhang, L.; Liu, H.; Ma, L. Z., Biological synthesis of highconductive pili in aerobic bacterium Pseudomonas aeruginosa. Appl. Microbiol. Biotechnol. 2019, 103, 1535-1544.

387 22. Pontrelli, S.; Chiu, T. Y.; E.I., L.; F.Y., C.; Chang, P.; J.C., L., Escherichia coli as a 388 host for metabolic engineering Metabolic Engineering 2018, 50, 16-46.

389 23. K.R., C.; J.H., S.; J.S., C.; D., Y.; S.Y., L., Systems metabolic engineering of Escherichia coli. EcoSal Plus 2016, 2016, doi:10.1128/ ecosalplus.ESP-0010-2015.

24. Wals, K.; Ovaa, H., Unnatural amino acid incorporation in E. coli: current and future applications in the design of therapeutic proteins. Front Chem 2:15. Front Chem 2014, 2, 15.

25. Smolskaya, S.; Andreev, Y. A., Site-specific incorporation of unnatural amino acids into Escherichia coli recombinant protein: methodology development and recent achievement. Biomolecules 2019, 9, 255. reconstitution of the type IVa pilus assembly system from enterohaemorrhagic Escherichia coli. Molecular Microbiology 2019, 111, 732-749. 27. Vargas, M.; Malvankar, N. S.; Tremblay, P.-L.; Leang, C.; Smith, J. A.; Patel, P.; Snoeyenbos-West, O.; Nevin, K. P.; Lovley, D. R., Aromatic amino acids required for pili conductivity and long-range extracellular electron transport in Geobacter sulfurreducens mBio 2013, 4, e00105-13. .

28. Liu, X.; Tremblay, P.-L.; Malvankar, N. S.; Nevin, K. P.; Lovley, D. R.; Vargas, M., A Geobacter sulfurreducens strain expressing Pseudomonas aeruginosa type IV pili localizes OmcS on pili but Is deficient in Fe(III) oxide reduction and current production. Appl Environ Microbiol 2014, 80, 1219-1224. Smith, J. A.; Snoeyenbos-West, O. L.; Franks, A. E.; Tuominen, M. T.; Lovley, D. R., The low conductivity of Geobacter uraniireducens pili suggests a diversity of extracellular electron transfer mechanisms in the genus Geobacter. Frontiers in microbiology 2016, 7, 980. derived from the trp and lac promoters Proc Natl Acad Sci U S A 1983, 80, 21-25. 
413 31. Datsenko, K. A.; Wanner, B. L., One-step inactivation of chromo- somal genes in 414 Escherichia coli K-12 using PCR products. Proc Natl Acad Sci U S A 2000, 97, 6640-6645.

415 32. Baba, T.; Ara, T.; Hasegawa, M.; Takai, Y.; Okumura, Y.; Baba, M.; Datsenko, K. 416 A.; Tomita, M.; Wanner, B. L.; Mor, i. H., Construction of Escherichia coli K-12 in-frame, 417 single-gene knockout mutants: the Keio collection. Molecular Systems Biology 2006, 2, 1-11.

418 33. Ueki, T.; Nevin, K. P.; Woodard, T. L.; Lovley, D. R., Genetic switches and related 419 tools for controlling gene expression and electrical outputs of Geobacter sulfurreducens. J. Ind. 420 Microbiol. Biotechnol. 2016, 43, 1561-1575.

421 34. Miller, J. H., Experiments in molecular genetics. Cold Spring Harbor Press: New York, 4221972.

423 35. Dehio, M.; Knorre, A.; Lanz, C.; Dehio, C., Construction of versatile high-level 424 expression vectors for Bartonella henselae and the use of green fluorescent protein as a new 425 expression marker. Gene 1998, 215, 223-229. 